\title{
New technics for removal of intradural spinal tumours
}

\author{
R.E. Rizea ${ }^{1,2}$, Karina Lidia Gheorghita ${ }^{2}$, \\ M.V. Salaceanu ${ }^{3}$, A.V. Ciurea ${ }^{4}$ \\ 1 Neurosurgery Department, "Bagdasar-Arseni" Emergency Neurosurgery \\ Hospital, Bucharest, ROMANIA \\ 2 "Carol Davila" University of Medicine and Pharmacy, Bucharest, RomanIA \\ 3 Neurosurgery Department, Sibiu Emergency Hospital, Sibiu, RomanIA \\ 4 Neurosurgery Department, Sanador Hospital, Bucharest, RomanIA
}

\begin{abstract}
Introduction. Neuronavigation is a computer-assisted technology based on pre- and intraoperative images that permit neurosurgeons to have a better approach of the brain and intradural spinal tumors. The neuronavigation systems have been a significant progress in neurosurgery. These systems allow neurosurgeons to evaluate surgical risks, select the best interventional method, localize better the tumors in order to improve the accuracy of the resection and decide on the optimal trajectory for the surgical procedure, resulting in decreased patient morbidity and mortality.

Material: Spinal cord tumors are rare and uncommon lesions. Their growth result in compression of the spinal cord, which can cause severe neurologic deficits such as limb dysfunction, motor and sensation loss with the possibility of leading to death. We present o short report of a study publicated by Stefini et al. in 2018 regarding the use of neuronavigation for removal of intradural spinal tumors.

Conclusion: The benefits of using neuronavigation in resection of the intradural spinal tumors include decreased risk of bad localization of the tumor, minimal invasive surgery technique and reduction of bone removal.
\end{abstract}

\section{INTRODUCTION}

Neuronavigation is a computer-assisted technology used by neurosurgeons in resection of the tumours to evaluate surgical risks, select the best interventional method, localize better the tumours in order to improve the accuracy of the resection and decide on the optimal trajectory for the surgical procedures. The technique is based on preand intraoperative images that permit neurosurgeons to have a better approach of the brain and intradural spinal tumours. The neuronavigation systems have been a significant progress in neurosurgery, their use resulted in decreased patient morbidity and mortality [1-4].

\section{MATERIAL}

The use of neuronavigation for the removal of spinal tumours has been described since 1990's, with good detailed notes in 2000 by Haberland

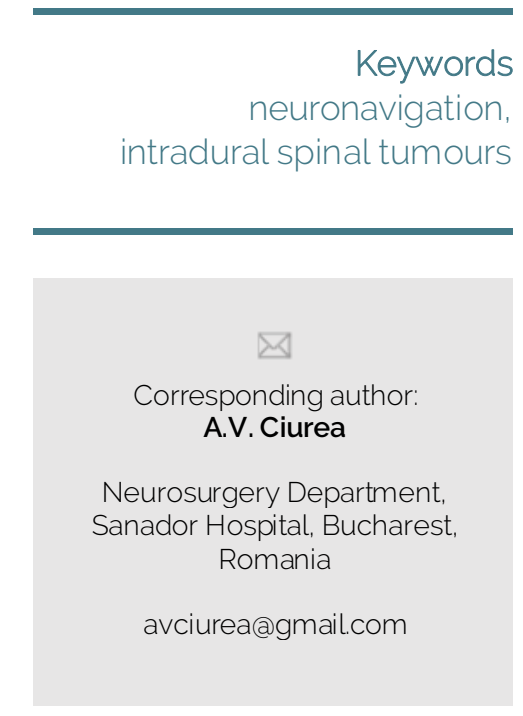

Copyright and usage. This is an Open Access article, distributed under the terms of the Creative commons Attribution Non-Commercial No Derivatives License (https://creativecommons org/licenses/by-nc-nd/4.0/) which permits noncommercial re-use, distribution, and reproduction in any medium, provided the original work is unaltered and is properly cited.

The written permission of the Romanian Society of Neurosurgery must be obtained for commercial re-use or in order to create a derivative work.

ISSN online 2344-4959 (C) Romanian Society of Neurosurgery

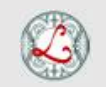

First published March 2019 by London Academic Publishing www.lapub.co.uk 
et al. $[1,2]$. Nowadays, spinal navigation is used to create 3D images in order to position the pedicle screws. A good microsurgical resection of spinal intradural tumours needs an accurate hemilaminectomy or laminectomy, that sometimes can be more excessive than necessary and leads to chronic pain and acquired deformities of the spine [3,4]. In 2018, Stefini et al. described the for first time the possibility to use the merging of the 3D fluoro images obtained intraoperative with spinal navigation with MRI preoperative images, in order to make a better approach of the spinal intradural or intramedullary tumours [3,5-7].

Stefini et al. published in 2018 a report regarding the use of neuronavigation for removal of intradural spinal tumours. They made a study between January and July 2016 that included 10 navigated procedures for intradural spinal tumours with the technique of merging 3D fluoro images obtained intraoperative with spinal navigation with MRI preoperative images. Initially, all the patients underwent contrastenhanced MRI with volumetric acquisitions and after that there were obtained the 3D fluoro images with neuronavigation. All these images were merged automatically or manually, verified if the association was correctly done on the sagittal, coronal and axial planes and used to perform all the steps of the required procedure using the navigated probes. The studied patients had all completed the fusion procedures, there were no errors detected and the intervention lasted about 20 min for each person [3].

\section{DISCUSSION}

Intraoperative navigation, considered an essential tool for cranial surgery, became in the last years an advantageous technique for spinal surgery in degenerative disc disease, spondylolisthesis, tumours and traumatic lesions. It reduces the neurological complications and system failure for the patient and the radiation exposure for the neurosurgical team $[3,8]$. The idea of merging the 3D fluoro images with the volumetric-enhanced MRI images made spinal navigation more accurate. This procedure resolved also problems like the centering of the tumours with a good indication of the precise level of the lesion, length of the skin incision, muscle strip and extent of bone removal, incidence of instability after the surgery, blood loss and postoperative pain $[3,9]$.

The technique was found very useful in case of small and intramedullary tumours, especially for the thoracic lesions because it reduces the need of radiation. The limitation of the procedure is that this it was not studied extensively $[3,10]$.

\section{CONCLUSIONS}

In our opinion this technique provides numerous advantages and it is simple to learn by the neurosurgeons. Intradural spinal tumours resection using neuronavigation is a minimal invasive technique that has numerous benefits including decreased risk of bad localization of the tumour, minimal invasive surgery technique and reduction of bone removal. The use of neuronavigation is in continuous progress of research that promises to be an excellent neurosurgery tool.

\section{REFERENCES}

1. Haberland N., Ebmeier K., Hliscs R. et al., J Cancer Res Clin Oncol (2000) 126: 529. https://doi.org/10.1007/s004320000122

2. Galloway RLJr, Maciunas RJ, Edwards CA. Interactive imageguided neurosurgery. IEEETransBiomedEng. 1992;39(12): 1226-1231.

3. Stefini R., Peron S., Mandelli J., Bianchini E., Roccucci P.; Intraoperative Spinal Navigation for the Removal of Intradural Tumors: Technical Notes, Operative Neurosurgery, Volume 15, Issue 1, 1 July 2018, Pages 5459, https://doi.org/10.1093/ons/opx179.

4. Bresnahan L, Ogden AT, Natarajan RN, Fessler RG. A biomechanical evaluation of graded posterior element removal for treatment of lumbar stenosis: comparison of a minimally invasive approach with two standard laminectomy techniques. Spine. 2009;34(1):17-23.

5. Ling JM, Dinesh SK, Pang BC, Chen MW, Lim HL, Louange DT. Routine spinal navigation for thoraco-lumbar pedicle screw insertion using the O-arm three-dimensional imaging system improves placement accuracy. J Clin Neurosci. 2014;21(3):493-498.

6. Roccucci P, Peron S, Minotti MR, Stefini R. Real-time navigation in spinal surgery: what has it changed in surgical practice? [published online ahead of print May4,2016] JNeurosurgSci.2016.

7. Vande Kelft E, Costa F, Vander Planken D, Schils F. A prospective multicenter registry on the accuracy of pedicle screw placement in the thoracic, lumbar, and sacral levels with the use of the O-arm imaging system and Stealth Station Navigation. Spine.2012;37(25):1580-1587.

8. Gebhard FT, Kraus MD, Schneider E, Liener UC, Kinzi L, Arand M. Does computer-assisted spine surgery reduce intraoperative radiation doses? Spine. 2006;31(17):20242027.

9. Mannion RJ, Nowitzke AM, Efendy J, Wood MJ. Safety and efficacy of intradural extramedullary spinal tumor removal 
using a minimally invasive approach. Neurosurgery.201;68 (1SupplOperative):208-216.

10. Neil W.D. Thomas, John Sinclair, Image-Guided Neurosurgery: History and Current Clinical Applications, Journal of Medical Imaging and Radiation Sciences, Volume
46, Issue 3, 2015, Pages 331-342, ISSN 1939-8654, https://doi.org/10.1016/j.jmir.2015.06.003.

(http://www.sciencedirect.com/science/article/pii/S193986 5415001903) 